\title{
A first-principles study of
}

hydrogen surface coverage on

$\delta$-Pu (100), (111), and (110) surfaces

\section{Ryan Gotchy Mullen ${ }^{1}$ and Nir Goldman ${ }^{1}$}

Physical and Life Sciences Directorate, Lawrence Livermore National Laboratory, Livermore, CA $94550 U_{S A}{ }^{\text {a) }}$

(Dated: 23 July 2021)

Hydriding corrosion of plutonium leads to surface cracking, pitting, and ultimately structural failure. Laboratory experiments demonstrate that hydriding begins on the surface or near subsurface of plutonium. However there has not yet been a systematic evaluation of hydrogen surface coverage on plutonium. In this work, we compute the surface energies of the low facet surfaces of face-centered cubic $\delta$-Pu. The adsorption free energies of expected hydrogen structures at low and high coverage are presented, along with the likely progression for filling sites as the $\mathrm{H}_{2}$ partial pressure increases. Implications for near-equilibrium pressure hydride nucleation and non-equilibrium millibar pressure hydriding are discussed.

${ }^{a)}$ Electronic mail: mullen10@llnl.gov 


\section{INTRODUCTION}

Plutonium's unique material properties make it useful as a heat source in power plants and in radioisotope thermoelectric generators. ${ }^{1}$ However, plutonium is highly reactive and will corrode if exposed to atmospheric gases, even in trace amounts. Hydriding in face-centered cubic (fcc) $\delta$-Pu occurs if the hydrogen concentration exceeds a temperature-dependent solubility limit. As it incorporates hydrogen to form plutonium dihydride $\left(\mathrm{PuH}_{2}\right)$, the plutonium lattice expands by approximately 60 volume \%, causing cracks and fissures in the plutonium surface. Moreover, $\mathrm{PuH}_{2}$ is pyrophoric, catalyzing rapid, violent exothermic oxidation. As a result, uncovering the mechanisms by which plutonium corrodes can improve safety regulations and eliminate toxic waste.

Conducting experiments with plutonium is difficult due to its toxicity and radioactivity. In addition, the complex phase diagram of plutonium makes it difficult to grow the single crystal materials necessary to characterize surface properties such as the surface energy. In particular, hydriding experiments are difficult since a $\mathrm{PuO}_{2}$ layer of nonuniform thickness is present on plutonium surfaces that have been exposed to air, providing variable protection to the underlying plutonium and introducing an element of randomness into the observed hydriding induction times. ${ }^{2}$ Lastly, experiments alone cannot provide a detailed atomicresolution hydriding mechanism nor identify ultimately how it can be controlled.

Quantum simulation methods, such as density functional theory (DFT), are necessary to model the bond breaking inherent in hydrogen dissociation and adsorption. ${ }^{3-6}$ DFT remains one of the most popular modeling methods in condensed matter physics, computational chemistry, and materials science, and has been widely used in studies of the physical and structural properties of $\delta$-Pu (e.g., Refs. 7,8). While a comprehensive explanation of plutonium's magnetism has yet to be offered, plutonium does not exhibit a bulk magnetic moment $^{9}$ and anti-ferromagnetic (AFM) models yield structural properties comparable to experiments. ${ }^{10}$

Surface hydriding studies are generally framed in terms of low (single molecule per surface unit) vs. high (nearing full monolayer) coverage. Low coverage conditions will be expected at hydrogen partial pressures $P_{H_{2}}$ well below the equilibrium pressure $P_{H_{2}}^{e q}, P_{H_{2}} \ll P_{H_{2}}^{e q}$. Previous DFT studies of adsorption of a single $\mathrm{H}$ atom or $\mathrm{H}_{2}$ molecule on clean (100) and (111) surfaces of $\delta$-Pu have all been conducted at low coverage conditions. ${ }^{3-6,11-13}$ High 
surface coverage will be expected when $P_{H_{2}} \gg P_{H_{2}}^{e q}$. The equilibrium pressure $P_{H_{2}}^{e q}$ at room temperature, has not been experimentally verified but was recently predicted from simulations to be $\mathcal{O}\left(10^{-19}\right)$ bar. ${ }^{14}$ Even very small pressures (e.g. 1 mbar) will exceed $P_{H_{2}}^{e q}$ by many orders of magnitude resulting in rapid adsorption and, unless the surface has a protective layer of $\mathrm{PuO}_{2}$, barrierless hydriding. Immediate hydriding at millibar pressures has been observed by experiments. ${ }^{2,15-18}$ We should therefore anticipate that under real world conditions, adsorption onto the surface from the gas phase may occur more quickly than surface adatoms can burrow from the surface into the bulk. However, to date there have been few systematic studies of Pu surface hydriding including determination of initial mechanisms as well as studies of middle-ranged surface coverage and how hydrogen can accumulate on a given facet.

In the present work, we use DFT calculations to determine hydrogen adsorption energies ranging from low to high surface coverage on low-index facets on $\delta$-Pu. We first use a simple two-state model to discuss relevant hydrogen surfaces coverages in terms of adsorption energetics when $P_{H_{2}} \approx P_{H_{2}}^{e q}$. This simple model helps place bounds on the numbers and types of adsorptions and their resulting energies that are likely relevant to initiating surface hydriding on different crystalline facets. We quantify bulk absorption of hydrogen in $\delta$-Pu and $\mathrm{PuH}_{2}$ at varying hydrogen concentrations, including the formation of point defects in $\mathrm{PuH}_{2}$. We then use DFT calculations to evaluate the surface energies of the (100), (111), and (110) surfaces to establish the relative abundance of each surface in polycrystalline materials. We explore three AFM geometries for each crystalline facet by alternating magnetic spins along (100), (111), and (110) planes. These results are also discussed in terms of the thermodynamic stability and morphologies of different nanocrystals. ${ }^{19}$ For the lowest energy AFM configuration of each surface, we next compute the adsorption energies of anticipated hydrogen structures with increasing surface coverage and compare to bulk absorption energies. Our efforts help determine an understanding of how increasing coverage affects subsequent hydrogen adsorption on the various surfaces of $\delta$-Pu and will provide insight into possible hydriding mechanisms observed in the bulk. 


\section{COMPUTATIONAL DETAILS}

Within DFT, $f$-electron correlations in bulk plutonium and plutonium hydride can be accounted for using either an on-site Coulomb repulsion (e.g. GGA+U) or orbital polarization, while the magnetic state can be computed through spin polarization (SP) or spin-orbit coupling (SOC) calculations. ${ }^{20-31}$ While spin-orbit coupling (SOC) is essential to compute material properties of $\mathrm{PuO}_{2},{ }^{25,32,33}$ it is less critical in simulating $\mathrm{H}-\mathrm{Pu}$ interactions in either the surface or bulk. ${ }^{25,29} \mathrm{SP}$ computed energies of low-index plutonium surfaces differ from SOC computed surface energies by only $7 \% .{ }^{8}$ In addition, adsorption energies of isolated hydrogen atoms on the (100) and (111) surfaces of plutonium differ by only $-0.03 \mathrm{eV}$ on average when computed with SP vs. SOC. ${ }^{6}$ As a result, we choose to employ the less computationally intensive GGA $+\mathrm{U}$ and SP methods in this work.

All DFT calculations discussed here were performed with the VASP code $^{34-36}$ using projector-augmented wave function (PAW) pseudopotentials ${ }^{37,38}$ and the Perdew, Burke, and Ernzerhof exchange correlation functional (PBE). ${ }^{39}$ The cutoff energy for the planewave expansion was set to $500 \mathrm{eV}$ and the wave function was converged to within $10^{-5} \mathrm{eV}$. Partial occupancies of the electronic states were set with a fourth-order Methfessel-Paxton smearing ${ }^{40}$ with a width of $0.3 \mathrm{eV}$.

Bulk $\delta$-Pu and $\mathrm{PuH}_{2}$ were modeled using 32 plutonium atoms and periodic boundary conditions. The magnetic moments of $\mathrm{Pu}$ atoms were treated using collinear SP and were alternated along (100) planes to give antiferromagnetic (AFM) configurations. A $5 \times 5 \times 5$ Monkhorst-Pack mesh ${ }^{41}$ was used for all bulk calculations, whereas we used a mesh of $5 \times 5 \times 1$ for our surface calculations, with the value of one aligned with the surface normal. We have used the GGA $+\mathrm{U}$ approach of Dudarev et al. ${ }^{42}$ as implemented in VASP. The value of the effective on-site Hubbard correction was $U-J=1.3 \mathrm{eV}(U=2.05 \mathrm{eV}$ and $J=0.75 \mathrm{eV})$ for $\delta-\mathrm{Pu}^{6}$ and $U-J=2.1 \mathrm{eV}(U=2.85 \mathrm{eV}$ and $J=0.75 \mathrm{eV})$ for $\mathrm{PuH}_{2} \cdot{ }^{14}$ Hydrogen atoms were placed in octahedral or tetrahedral sites, after which ionic positions were relaxed using the conjugate gradient algorithm until forces were less than $0.05 \mathrm{eV} / \AA$. Cell size and shape were optimized under zero pressure.

For our adsorption energy calculations, hydrogen atoms were added to a given surface adsorption site and allowed to relax normal to the surface. We constructed low coverage structures that have hydrogen atoms adsorbed at isolated sites and as dimers with two atoms 
occupying neighboring sites. High coverage structures have all surface sites filled and variations with single site or double site vacancies. As possible sets of intermediate structures, we filled all sites of one type and included variations with single or double vacancies as well as single or double occupancy of the other site type.

Slabs of $\delta$-Pu were created with exposed (100), (111), and (110) surfaces. Each slab consists of 4 layers of plutonium atoms with $30 \AA$ of vacuum. Atoms in the bottom two layers were held fixed in place, while atoms in the top two layers were allowed to relax. Magnetic spins alternate along (100), (111), or (110) planes to give AFM configurations.

\section{TWO-STATE MODEL}

We consider surface coverge under equilibrium conditions using a simplified two-state model. For a system of $N_{H}$ hydrogen atoms partitioned between $N^{\text {bulk }}$ bulk sites and $N^{\text {surf }}$ surface sites, the surface coverage is

$$
\theta=N_{H}^{\text {surf }} / N^{\text {surf }}
$$

where $N_{H}^{\text {surf }}$ is the number of hydrogen atoms adsorbed to surface sites. The absorption energy at each bulk (surface) site is $E^{\text {bulk }}\left(E^{\text {surf }}\right)$, and the difference between these energies is $\Delta E=E^{\text {surf }}-E^{\text {bulk }}$. The energy of an $N_{H}^{\text {surf }}$ microstate is therefore $E=N_{H}^{\text {surf }} \Delta E$, ignoring constant terms.

At constant temperature $T$, the average number of atoms adsorbed on surface sites is

$$
\left\langle N_{H}^{\text {surf }}\right\rangle=\sum_{E} \frac{e^{-\beta E} \Omega(E) N_{H}^{\text {surf }}}{Q(T)}
$$

where $\Omega$ is the microcanonical density of states, $Q$ is the canonical partition function, and the dependence of $\Omega$ and $Q$ on $N_{H}, N^{b u l k}$, and $N^{\text {surf }}$ have been omitted for clarity. The density of states $\Omega(E)$ is the product of probabilities for choosing $N_{H}^{\text {surf }}$ sites from the $N^{\text {surf }}$ possibilities and for choosing $N_{H}^{b u l k}$ sites from the $N^{b u l k}$ possibilities

$$
\Omega=\frac{N^{\text {surf }} !}{N_{H}^{\text {surf }} !\left(N^{\text {surf }}-N_{H}^{\text {surf }}\right) !} \frac{N^{\text {bulk }} !}{N_{H}^{\text {bulk }} !\left(N^{\text {bulk }}-N_{H}^{\text {bulk }}\right) !}
$$

Here, the second probability related to $N_{H}^{b u l k}$ was computed via Sterling's approximation for the sake of simplicity. 
We consider slabs with $N^{\text {surf }}=100$ sites and $N^{\text {bulk }}$ ranging from $10^{4}-10^{7}$. The number of hydrogen atoms is set to $N_{H}=10^{-3} N^{\text {bulk }}$ to match the upper bound for $\mathrm{H}$ absorption in $\delta$-Pu at room temperature. ${ }^{14}$ At each system size, we then vary the value of $\Delta E$ from highly positive values (corresponding to strongly favorable bulk absorption) to highly negative (corresponding to strongly favored surface adsorption) in order to determine the range of $\Delta E$ that will permit high surface coverage.

Fig. 1 shows surface coverage $\theta$ as a function of $\Delta E$. For $\Delta E=0$, surface sites will be populated in the same proportion as the bulk, $\theta=N_{H}^{b u l k} / N^{b u l k}$. As the surface adsorption energy increases ( $\Delta E$ becomes more positive), surface coverage drops exponentially. As the adsorption energy decreases ( $\Delta E$ becomes more negative), surface coverage increases but begins to plateau as maximum capacity is reached. For the smallest system size, $N^{b u l k}$ $=10,000$, there are only $N_{H}=10$ atoms so the maximum surface coverage is $10 \%$. For systems with $N^{\text {bulk }}>1$ million sites, $N_{H}>N^{\text {surf }}$ and we see that the two-state model is not artificially constrained to low $\theta$. For $\Delta E=-10 k_{B} T$, the converged surface coverage is $\theta=96 \%$.

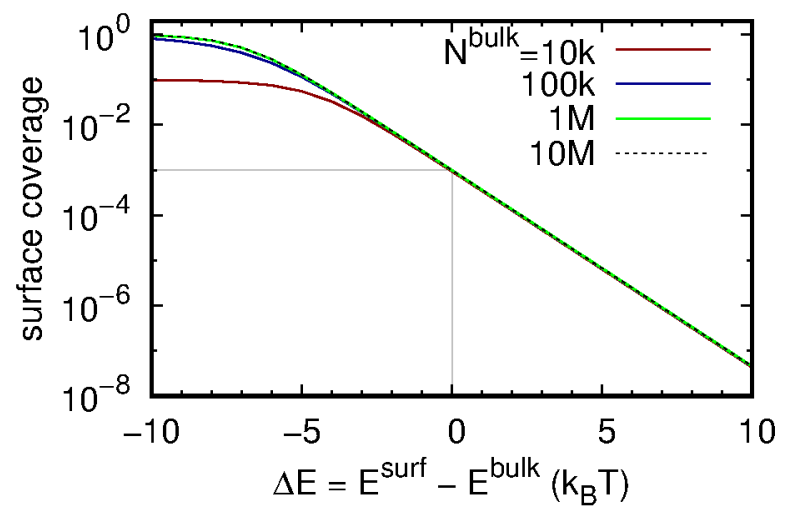

FIG. 1. Surface coverage $\theta$ as a function of $\Delta E$ for a two-state slab model with $N^{\text {surf }}=100$ sites and varying numbers of bulk sites.

This simplified two-state model of adsorption illustrates convenient guidelines to evaluate surface coverage at equilibrium. Sites with adsorption energies within $\pm 2 k_{B} T$ of the bulk absorption energy will be populated at approximately the bulk ratio, $N_{H} / N_{P u}$. Surface sites that are $10 k_{B} T$ higher in energy than bulk adsorption will have negligible population. Sites that are $10 k_{B} T$ lower in energy will be entirely filled. We can thus use $\pm 10 k_{B} T$ as an 
approximate bound for gauging how absorbed hydrogen atoms partition between surface and bulk sites. The first step in applying this framework to $\mathrm{H}$ surface coverage on $\delta$-Pu is to compute bulk absorption energies, which is where we now turn.

\section{RESULTS}

\section{A. Hydrogen absorption in bulk $\delta$-Pu and $\mathrm{PuH}_{2}$}

The energy released as hydrogen atoms are absorbed into bulk $\delta$-Pu from the gas phase is

$$
\Delta E_{i}^{\text {bulk }}=E_{i}^{\text {bulk }}-N_{P u} e_{P u}^{\text {bulk }}-N_{H} e_{H}
$$

where $e_{P u}^{b u l k}$ is the energy of a plutonium atom in a defect-free fcc lattice, $e_{H}$ is the energy of a hydrogen atom in a gas-phase hydrogen molecule, and $N_{P u}$ and $N_{H}$ are the number of plutonium and hydrogen atoms in configuration $i$, respectively. The absorption energy reveals that it is energetically favorable for an isolated hydrogen atom in bulk $\delta$-Pu to absorb at an $\mathrm{O}$ site $\left(\Delta E_{O 1}^{\text {bulk }}=-2.92 \mathrm{eV}\right)$ rather than at a $\mathrm{T}$ site $\left(\Delta E_{T 1}^{\text {bulk }}=-2.71 \mathrm{eV}\right)$. The difference $\Delta E_{T 1}^{\text {bulk }}-\Delta E_{O 1}^{\text {bulk }}=0.21 \mathrm{eV}\left(8 k_{B} T\right.$ at room temperature $)$ and the corresponding Boltzmann factor, $\exp (-8)$, shows that the fraction of absorbed hydrogen occupying $\mathrm{T}$ sites is $\mathcal{O}\left(10^{-4}\right)$.

There are three possible nearest neighbor $N_{H}=2$ clusters in bulk $\delta$-Pu: neighboring occupied $\mathrm{O}$ sites (O2), neighboring occupied $\mathrm{T}$ sites (T2), and an occupied $\mathrm{O}$ site next to an occupied T site (T1O1). The $\mathrm{O} 2$ absorption energy $\Delta E_{O 2}^{\text {bulk }}$ is $0.25 \mathrm{eV}\left(10 k_{B} T\right)$ greater than the energy of two independent $\mathrm{O} 1$ absorbates, $2 \Delta E_{O 1}^{\text {bulk }}$, so the equilibrium population of $\mathrm{O} 2$ configurations will be $\mathcal{O}\left(10^{-5}\right)$ of the absorbed hydrogen. The T1O1 and T2 absorption energies are even higher, $21 k_{B} T$ and $27 k_{B} T$ above the O1 absorption energy, respectively, indicating that the equilibrium population of either is negligible.

The preceding analysis demonstrates that $\Delta E_{O 1}^{b u l k}$ is a convenient reference for evaluating absorption energies. Normalizing $\Delta E_{i}^{\text {bulk }}$ by $N_{H}$ and subtracting the reference absorption energy $\Delta E_{O 1}^{\text {bulk } \text { yields }}$

$$
\Delta \Delta e_{i}^{\text {bulk }}=\frac{\Delta E_{i}^{\text {bulk }}}{N_{H}}-\Delta E_{O 1}^{\text {bulk }} .
$$

$\Delta \Delta e_{i}^{\text {bulk }}$ will be negative for configurations that are more stable than a set of $N_{H}$ atoms distributed across isolated $\mathrm{O}$ sites, and positive for configurations that are less stable. Fig. 2 
(lower part of left plot) shows $\Delta \Delta e_{i}^{b u l k}$ is positive for T1 and all $N_{H}=2$ configurations in $\delta-\mathrm{Pu}$.

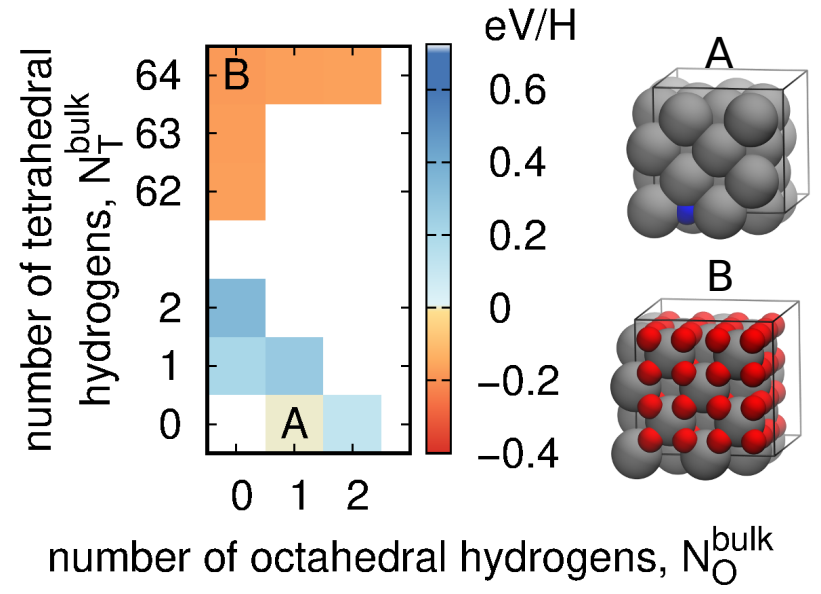

FIG. 2. (Left) Normalized absorption energy $\Delta \Delta e_{i}^{b u l k}$ for configurations in the $\delta$-Pu and $\mathrm{PuH}_{2}$ phases. (Right) Snapshots of the most energetically favorable DFT-optimized bulk configurations in the $\delta$-Pu (A) and $\mathrm{PuH}_{2}$ (B) phases. $\mathrm{Pu}$ atoms are grey, $\mathrm{H}$ atoms in $\mathrm{O}$ sites are blue, and $\mathrm{H}$ atoms in $\mathrm{T}$ sites are red.

We now perform a similar analysis on $\mathrm{PuH}_{2}$, where we can vary the number of $\mathrm{O}$ site hydrogens in a perfect crystal populated initially with $\mathrm{T}$ site hydrogen, only (i.e., T64). Fig. 2 (upper part of left plot) shows that all absorption configurations exhibit negative $\Delta \Delta e_{i}^{\text {bulk }}$ values, indicating that there is an energetic driving force for dilute $\mathrm{H}$ atoms to assemble and form a dihydride product. However, since for $\delta$-Pu absorption all $N_{H}=2$ configurations are higher in energy than O1 (single, dilute $\mathrm{H}$ absorption), there clearly are energetic barriers as well as entropic barriers to hydride nucleation.

We gain further insights into the structure of the $\mathrm{PuH}_{2}$ phase from the energetics of forming $\mathrm{T}$ site vacancies and $\mathrm{O}$ site interstitials. The defect-free $\mathrm{PuH}_{2}$ crystal has the lowest $\Delta \Delta e_{T 64}^{\text {bulk }}=-0.18 \mathrm{eV} / \mathrm{H}$. Compared to T64, T63 has a single $\mathrm{T}$ site vacancy. The vacancy formation energy

$$
E_{T}^{v a c}=\Delta E_{T 63}^{b u l k}-\Delta E_{T 64}^{b u l k}
$$

is $3.24 \mathrm{eV}$. Similarly, the energy released by an $\mathrm{O}$ site interstitial

$$
E_{O}^{i n t}=\Delta E_{T 64 O 1}^{b u l k}-\Delta E_{T 64}^{b u l k}
$$




\begin{tabular}{lrrr}
\hline & $(100)$ & $(111)$ & $(110)$ \\
\hline $\operatorname{AFM}(100)$ & 937 & 1107 & 1088 \\
$\operatorname{AFM}(111)$ & 1018 & 807 & 1052 \\
$\operatorname{AFM}(110)$ & 974 & 854 & 980 \\
$\operatorname{FM}$ & $1088^{a}$ & $970^{b}$ & - \\
\hline
\end{tabular}

${ }^{a}$ Ref. 8

${ }^{b}$ estimated from Ref. 7

TABLE I. Surface energies, in $\mathrm{mJ} / \mathrm{m}^{2}$, of unalloyed $\delta$-Pu

is $-2.50 \mathrm{eV}$. The net reaction energy of forming an $\mathrm{O}$ site interstitial and a $\mathrm{T}$ site vacancy (e.g., $\left.E_{T}^{v a c}+E_{O}^{i n t}\right)$ is $+0.74 \mathrm{eV}\left(29 k_{B} T\right)$. This in turn precludes this bulk reaction mechanism and indicates that $\mathrm{O}$ site interstitials in $\mathrm{PuH}_{2}$ are not created unless the $\mathrm{H}_{2}$ pressure is high enough to fill all $\mathrm{T}$ sites.

\section{B. $\delta$-Pu surface energies}

The surface energies of the (100), (111), and (110) surfaces of $\delta$-Pu were predicted by computing the DFT energies of Pu slabs. The surface energy is

$$
\gamma^{s}=\frac{E_{P u}^{s}-N_{P u} e_{P u}^{b u l k}}{2 A^{s}}
$$

where $E_{P u}^{s}$ is the DFT energy of a plutonium slab with two exposed $s$-oriented surfaces and $A^{s}$ is the exposed surface area. Surface energies are reported in Table I for three antiferromagnetic (AFM) geometries of each surface and compared to previous computations of ferromagnetic (FM) plutonium surfaces. Surface energies of the FM (111) surface with spin polarization have not been previously computed. However, we note that Wu et al. ${ }^{8}$ found that spin polarization lowered the computed (100) surface energy by $28 \%$. We can thus apply the same reduction to the non-spin-polarized (111) surface energy from Ray et al., ${ }^{7} 1342 \mathrm{~mJ} / \mathrm{m}^{2}$, to approximate the spin-polarized FM (111) surface energy.

We find that in general the AFM configurations have lower surface energies than the corresponding FM surfaces and that the surface energy is minimized by the AFM configuration with planes of magnetization that are parallel to the surface. As a result, we would 

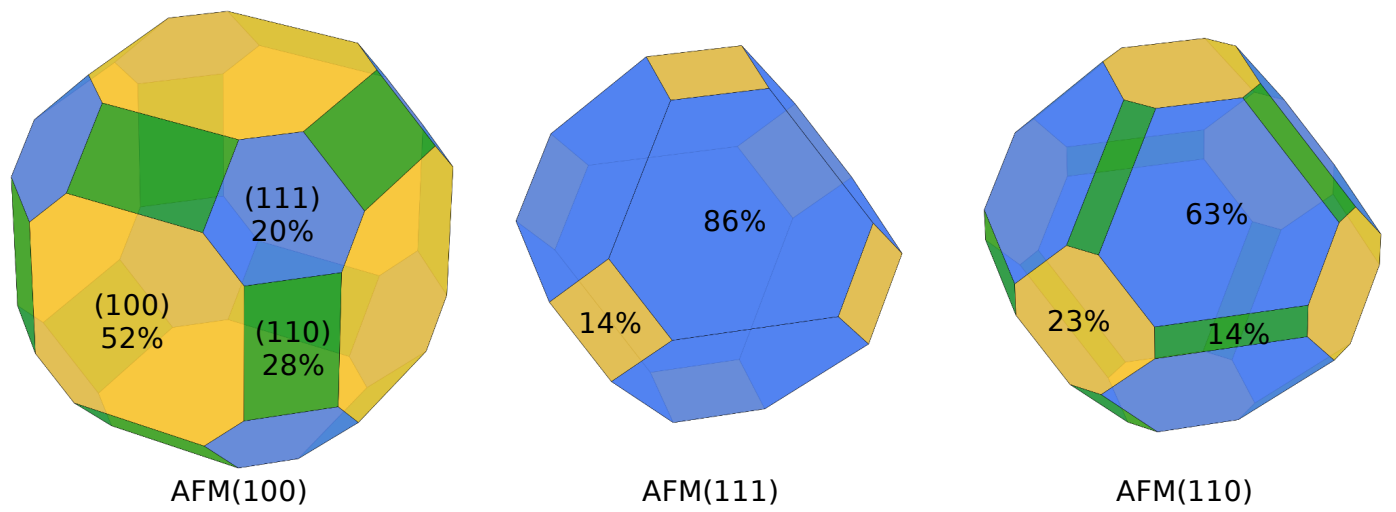

FIG. 3. Wulff constructions of $\delta$-Pu nanocrystals showing the relative surface area of the (100), (111), and (110) surfaces in yellow, blue, and green, respectively.

expect single crystal surfaces of $\delta$-Pu to have AFM geometries that parallel that particular surface. Real structures made from $\delta$-Pu will be polycrystalline with grain boundaries that may prevent such idealized alignment. To give a sense of the relative abundance of each surface orientation in polycrystalline materials, we present Wulff constructions of plutonium nanocrystals for each of the three AFM configurations considered (see Fig. 3). For $\operatorname{AFM}(100)$ nanoparticles, the lowest energy surface is the (100), accounting for $52 \%$ of its surface area. The remainder of the surface is apportioned between (110) surfaces and (111) surfaces. For $\operatorname{AFM}(111)$ nanoparticles, surface area is dominated by (111) surfaces $(86 \%)$ with the remainder being (100) surfaces. An $\operatorname{AFM}(110)$ nanocrystal surface is also predominantly (111) surfaces (63\%), but does exhibit non-negligible (110) surface area (14\%).

\section{C. (100) Surface monolayer formation}

On a surface of $\delta-\mathrm{Pu}$, the adsorption energy is

$$
\Delta E_{i}^{s}=E_{i}^{s}-E_{P u}^{s}-N_{H} e_{H}
$$

where $E_{i}^{s}$ is the DFT energy of a plutonium slab with $s$-oriented surfaces and with configuration $i$ of surface hydrogens. $\Delta \Delta e_{i}^{s}$ is defined similarly to Eq. 5

$$
\Delta \Delta e_{i}^{s}=\frac{\Delta E_{i}^{s}}{N_{H}}-\Delta E_{O 1}^{a b s}
$$

The (100) surface of $\delta$-Pu has two surface adsorption sites: hollow (H) sites and bridge (B) sites. The (100) slab considered here has 8 surface $\mathrm{Pu}$ atoms, and therefore $8 \mathrm{H}$ sites and 
$16 \mathrm{~B}$ sites. We find that isolated hydrogen atoms adsorb more favorably at hollow sites, $\Delta E_{H 1}^{(100)}=-3.08 \mathrm{eV}$, than at bridge sites, $\Delta E_{B 1}^{(100)}=-2.67 \mathrm{eV}$, consistent with previous results. 6

We now sequentially fill the $\mathrm{H}$ and $\mathrm{B}$ sites on the (100) surface and compute $\Delta \Delta e_{i}^{(100)}$ for each configuration. We quantify how hydrogen adsorption changes as a function of surface coverage by computing an adsorption energy grid, whereby $\mathrm{H}$ and $\mathrm{B}$ sites are monotonically populated with hydrogens. In doing so, we can determine the energetic requirements for surface monolayer coverage. Fig. 4 shows the per atom adsorption energies $\Delta \Delta e_{i}^{(100)}$ of tested structures in four quadrants: low-coverage (lower left corner), hollow-occupied (lower right corner), bridge-occupied (upper left corner), and high-coverage (upper right corner). We can identify the most favorable configurations at low and high surface coverage and trace the thermodynamic minimum energy path between them. At low surface coverage, the most favorable adsorption occurs at an isolated hollow site (see Fig. $4 \mathrm{C}$ ), $\Delta \Delta e_{H 1}^{(100)}=-0.16 \mathrm{eV} / \mathrm{H}$. At high surface coverage, the most favorable configuration has all $\mathrm{H}$ sites filled and a doublevacancy in B sites (see Fig. $4 \mathrm{~F}$ ), $\Delta \Delta e_{H 8 B 14}^{(100)}=0.40 \mathrm{eV} / \mathrm{H}$. The minimum energy path from low to high coverage can go through the hollow-occupied quadrant (see Fig. 4D) or through the bridge-occupied quadrant (see Fig. 4E). The H6 adsorption energy, $\Delta \Delta e_{H 6}^{(100)}=0.006 \mathrm{eV} / \mathrm{H}$, is intermediate to that of the low and high coverage while the H2B16 energy, $\Delta \Delta e_{H 2 B 16}^{(100)}=$ $0.62 \mathrm{eV} / \mathrm{H}$, exceeds that of the high coverage states. As a result, we expect that surface hydrogens fill $\mathrm{H}$ sites before starting to occupy B sites.

Normalized adsorption energies $\Delta \Delta e_{i}^{(100)}$ are shown as a function of a surface coverage in Fig. 5. In addition to the 23 configurations optimized for Fig. 4, we computed the energies of 20 configurations with surface hydrogens randomly distributed among the $\mathrm{H}$ and B sites. Importantly, the adsorption energies of the random structures are bounded on the low end by the path CDF shown in Fig. 4 (see also line CDF on Fig. 5) and on the high end by the cluster of bridge-occupied structures (see point E on Fig. 5). Up to surface coverages of $\theta \approx 1 / 3$, the lowest energy configurations only have occupied $\mathrm{H}$ sites and the adsorption energy is a weak function of coverage. At higher coverages, hydrogens have to occupy the higher energy B sites and the adsorption energy increases to approximately $0.4-0.5 \mathrm{eV} / \mathrm{H}$, exceeding the $10 k_{B} T$ threshold at $\theta \approx 0.65$. 

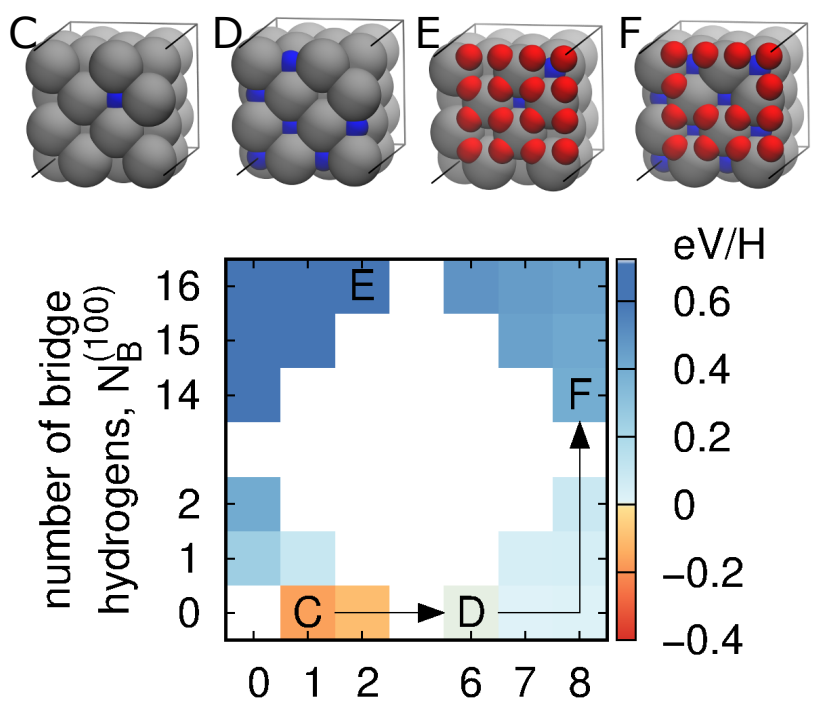

$\mathrm{eV} / \mathrm{H}$

0.6

0.4

0.2

0

$-0.2$

number of hollow hydrogens, $\mathrm{N}_{\mathrm{H}}^{(100)}$

FIG. 4. (Top) Snapshots of the most energetically favorable DFT-optimized configurations with hydrogen adsorbed to the (100) surface corresponding to each quadrant of coverage: low (C), hollowoccupied (D), bridge-occupied (E), high (F). Pu atoms are grey, $\mathrm{H}$ atoms in $\mathrm{H}$ sites are blue, and $\mathrm{H}$ atoms in $\mathrm{B}$ sites are red. (Bottom) Normalized adsorption energy for each configuration relative to the absorption energy of an isolated hydrogen atom in a bulk $\mathrm{O}$ site.

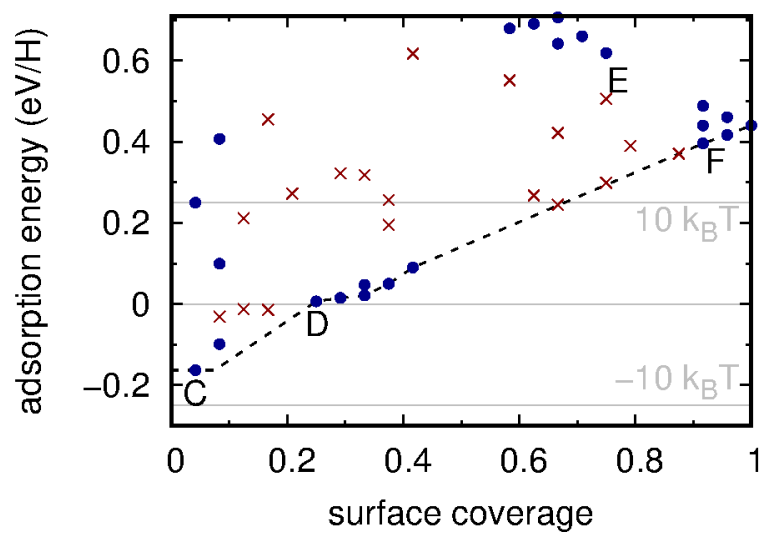

FIG. 5. Normalized adsorption energy as a function of surface coverage on the (100) surface for each configuration in Fig. $4(\bullet)$ and for configurations with randomly distributed hydrogen atoms $(\times)$ for comparison. Lines are intended as a guide to the eye. 


\section{D. (111) Surface monolayer formation}

On the (111) surface of $\delta$-Pu, hydrogen atoms can adsorb at fcc or hexagonal close-packed (hcp) sites, which differ only in the positioning of plutonium atoms in the first subsurface layer. If a hydrogen atom moves from a surface hcp site into the bulk, it will occupy a $\mathrm{T}$ site. If a hydrogen atom in a surface fcc site moves into the bulk, it will occupy an $\mathrm{O}$ site. The (111) slab used here has 8 surface $\mathrm{Pu}$ atoms, and therefore has 8 fcc and 8 hcp sites. We find that the adsorption energy of a single $\mathrm{H}$ atom at either site are indistinguishable, $\Delta E_{h c p 1}^{(111)}=-2.69 \mathrm{eV}$ versus $\Delta E_{f c c 1}^{(111)}=-2.68 \mathrm{eV}$, consistent with previous results. ${ }^{6}$

Our computed adsorption energy grid in Fig. 6 shows that the per atom adsorption energies of all configurations exhibit only a weak dependence on surface coverage. The lowest adsorption energy in each of the low-coverage, high-coverage, fcc-occupied, and hcpoccupied quadrants are all quite similar relative to bulk absorption, within a range of 0.085 $\mathrm{eV} / \mathrm{H}$. The lowest adsorption energy is observed in the fcc-occupied quadrant (see Fig. 6I), with a value of $\Delta \Delta e_{f c c 7}^{(111)}=0.19 \mathrm{eV} / \mathrm{H}$.

Random sampling in Fig. 7 shows that $\Delta \Delta e^{(111)}$ energies are relatively flat with surface coverage. Adsorption energies on (111) are minimized at moderate coverage in the range $0.3<\theta<0.5$ rather than at low coverage as on (100). Similar to the (100) surface, the energies of randomized structures are also bounded by the convex hull of structures examined for Fig. 6. Due to the similarity in energies for hcp and fcc sites, the (111) surface is likely to fill randomly with increasing $P_{H 2}$.

\section{E. (110) Surface monolayer formation}

The (110) surface is not as atomically smooth as the (100) and (111) surfaces. Hydrogen atoms can adsorb onto short-bridge (SB) sites on top of plutonium ridges, or onto hollow $(\mathrm{H})$, fcc, and long-bridge (LB) sites in the grooves between ridges. Unlike the (100) and (111) slabs which had 8-16 of each site, the (110) slab only has 4 LB, SB, or H sites. As a result, we cannot compute the adsorption energies of some hydrogen adsorption dimers or double vacancies since, for example, two hydrogen atoms in neighboring LB sites would fill the entire periodic valley. We will limit our analysis of (110) configurations to isolated hydrogen atoms, single vacancies, and dimers or double vacancies involving different types of 


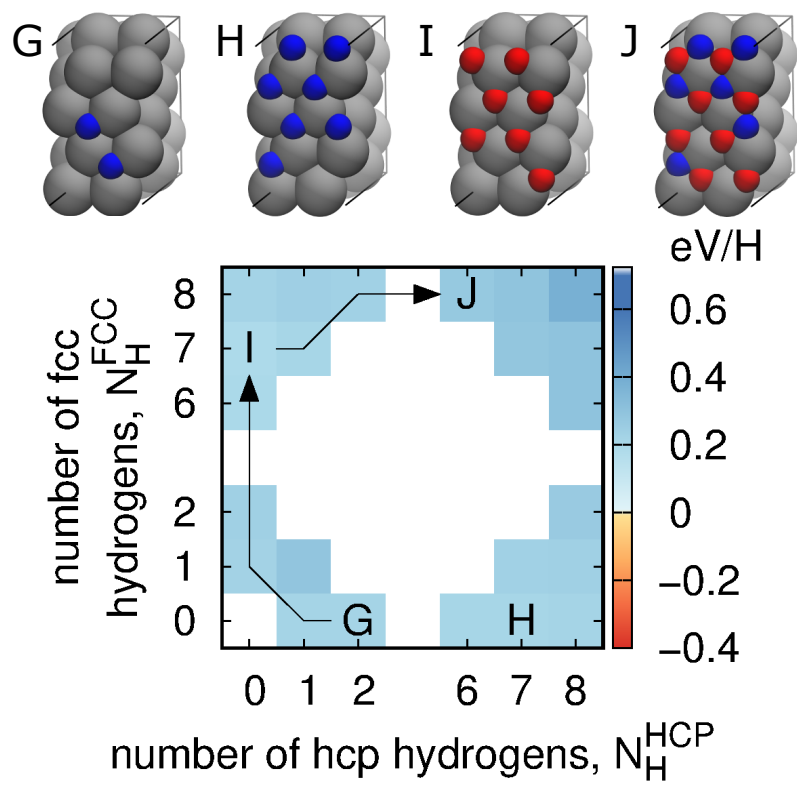

FIG. 6. (Top) Snapshots of the most energetically favorable DFT-optimized configurations with hydrogen adsorbed to the (111) surface corresponding to each quadrant of coverage: low $(\mathrm{G})$, hcpoccupied (H), fcc-occupied (I), high (J). Pu atoms are grey, $\mathrm{H}$ atoms in hcp sites are blue, and $\mathrm{H}$ atoms in fcc sites are red. (Bottom) Normalized adsorption energy for each configuration relative to the absorption energy of an isolated hydrogen atom in a bulk $\mathrm{O}$ site.

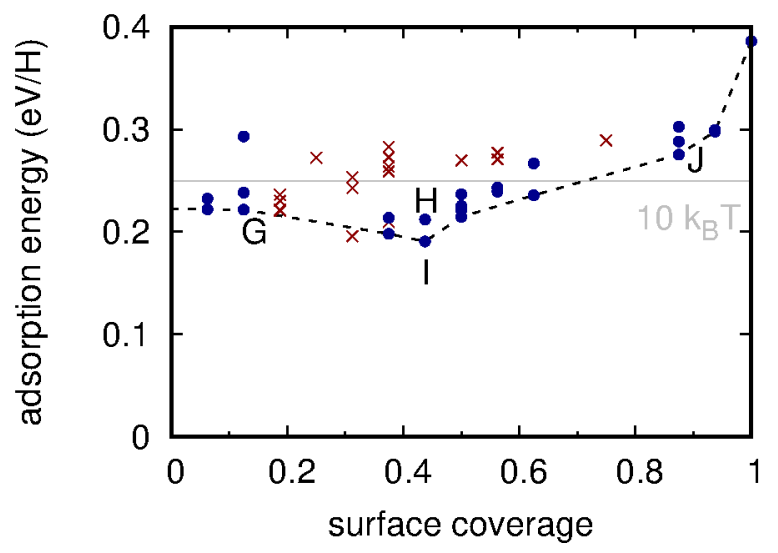

FIG. 7. Normalized adsorption energy as a function of surface coverage on the (111) surface for each configuration in Fig. $6(\bullet)$ and for configurations with randomly distributed hydrogen atoms $(\times)$ for comparison. Lines are intended as a guide to the eye. 


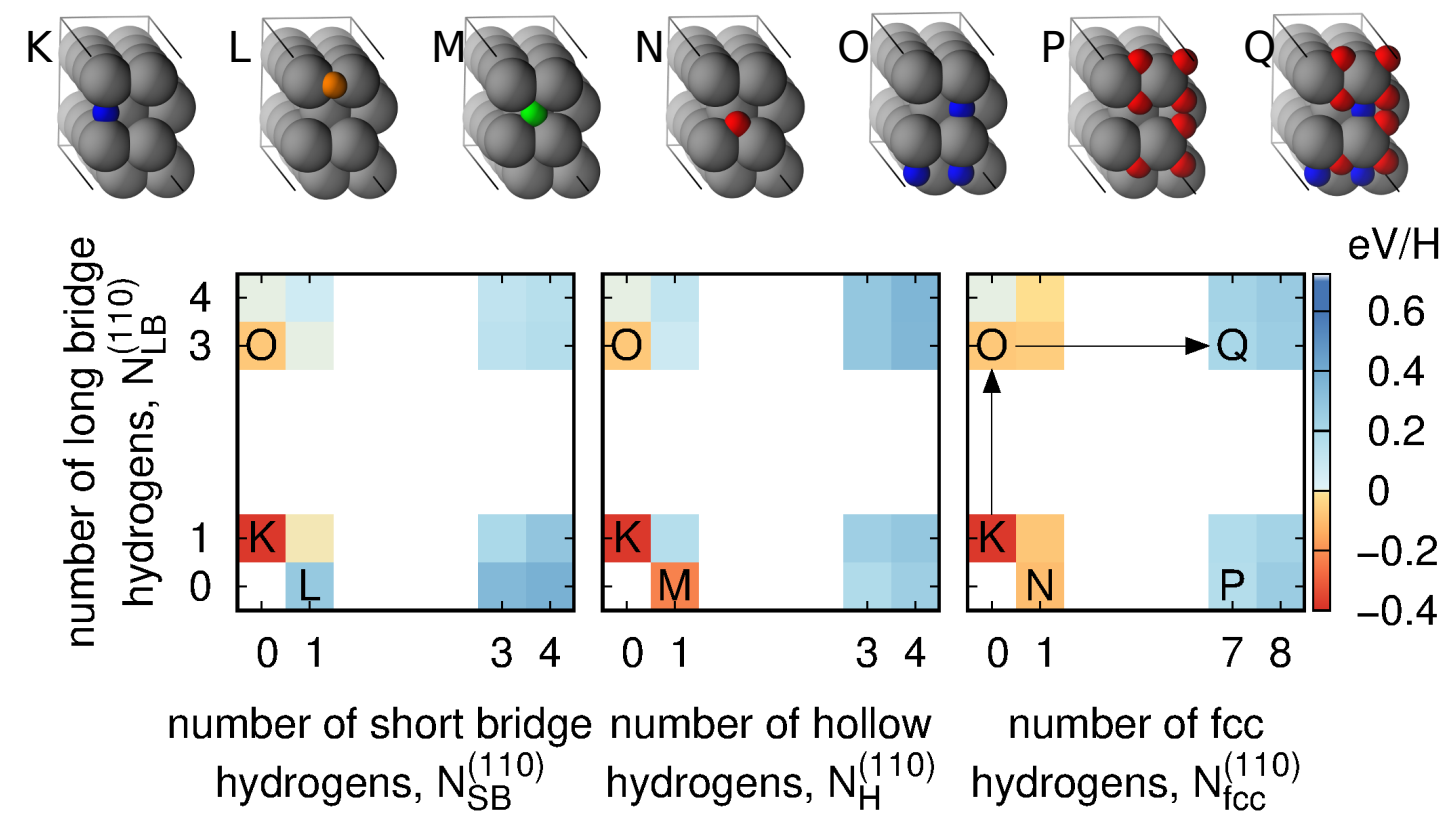

FIG. 8. (Top) Snapshots of select DFT-optimized configurations with hydrogen adsorbed to the (110) surface. Pu atoms are grey, $\mathrm{H}$ atoms in LB sites are blue, in SB sites are are orange, in $\mathrm{H}$ sites are green, and in fcc sites are red. (Bottom) Normalized adsorption energy for each configuration relative to the absorption energy of an isolated hydrogen atom in a bulk $\mathrm{O}$ site.

sites. Even still, the number of possible combinations of site types leads to a large number of configurations to evaluate. To make the problem tractable, we further restrict our analysis to LB-SB, LB-H, and LB-fcc pair combinations.

Fig. 8 shows that isolated hydrogen atoms adsorb more favorably than in the bulk at $\mathrm{LB}, \mathrm{H}$, and fcc sites. LB sites are so attractive (see Fig. $8 \mathrm{~K}$ ), $\Delta \Delta e_{L B 1}^{(110)}=-0.38 \mathrm{eV} / \mathrm{H}$ $\left(-15 k_{B} T / \mathrm{H}\right)$, that thermal energy is unlikely to dislodge adsorbed hydrogen atoms. Adsorption at an $\mathrm{H}$ site (see Fig. $8 \mathrm{M}$ ) is the next most negative, $\Delta \Delta e_{H 1}^{(110)}=-0.23 \mathrm{eV} / \mathrm{H}$ and is stabilized by the $\mathrm{Pu}$ ridges on either side which each move inward by $0.3 \AA$. The next lowest adsorbtion occurs at fcc sites (see Fig. $8 \mathrm{~N}$ ) and is only a few $k_{B} T$ more favorable than bulk adsorption, $\Delta \Delta e_{f c c 1}^{(110)}=-0.10 \mathrm{eV} / \mathrm{H}\left(-4 k_{B} T / \mathrm{H}\right)$. As on the (111) surface, a hydrogen in an (110) fcc site that burrows into the surface will land in an O site. Overall, unlike the (100) and (111) surfaces, in which the thermodynamic driving force directs hydrogen from the surfaces into the bulk, the (110) surface attracts hydrogen from the bulk.

Even though (110) is attractive to individual hydrogen atoms, adsorption dimer energeticsrelevant to adsorbing consecutive hydrogen molecules from the gas phase - are more compli- 
cated. Possible repulsion or attraction between adsorbed hydrogen atoms can be detected by defining the interaction energy

$$
\Delta \Delta E_{i j}^{s, i n t}=\Delta E_{i j}^{s}-N_{i}^{s} \Delta E_{i}^{s}-N_{j}^{s} \Delta E_{j}^{s}
$$

where $i$ and $j$ are different hydrogen configurations. Hydrogen atoms in an LB1H1 dimer are $1.8 \AA$ apart with an interaction energy $\Delta \Delta E_{L B 1 H 1}^{(110), \text { int }}=0.93 \mathrm{eV}$. In other words, hydrogen atoms adsorbed on nearest neighbor LB and $\mathrm{H}$ sites are nearly $1 \mathrm{eV}$ higher in energy than hydrogen adsorbed on distant LB and H sites. In LB1fcc1 and LB1SB1, the hydrogen atoms are 2.1 and $3.4 \AA$ apart with $\Delta \Delta E^{\text {int }}$ of 0.33 and $0.10 \mathrm{eV}$, respectively, showing that the repulsive interaction decays with increasing distance.

As surface coverage on (110) increases, we consider surfaces that have all or all but one of a single site type filled. In this limit, LB sites are the only ones that still have more favorable adsorption than the bulk. If all but one LB site are filled (see Fig. 8O), $\Delta \Delta e_{L B 3}^{(110)}$ $=-0.07 \mathrm{eV} / \mathrm{H}$. In contrast, $\Delta \Delta e$ becomes positive as either all $\mathrm{H}$ or fcc sites are filled. When all $\mathrm{H}$ sites are occupied, the $\mathrm{Pu}$ ridges are pulled equally towards the hydrogen on either side and so cannot move to stabilize adsorbates in one valley without destabilizing adsorbates in the other. When all fcc sites are occupied, each hydrogen will be within $2 \AA$ of another, suggesting that hydrogen-hydrogen repulsion raises the energy of these configurations. Starting from an LB3 configuration, adding a hydrogen to an SB or H site results in a positive $\Delta \Delta e$, while adding one to an fcc site results in a negative $\Delta \Delta e$. As a result, we next consider the adsorption energy as a function of surface coverage considering only the LB and fcc sites.

Fig. 9 shows normalized adsorption energies $\Delta \Delta e_{i}^{(110)}$ as a function of coverage of LB and fcc sites. In addition to the 15 LB-fcc configurations evaluated for Fig. 8, we computed adsorption energies for 20 configurations with surface hydrogens randomly distributed among the LB and fcc sites. At low coverage, adsorbed hydrogens can occupy independent LB sites. As the number of vacant LB sites decreases, there is a steep increase in adsorbtion energy. After the LB sites are filled, hydrogens occupy fcc sites and the adsorption energy increases more shallowly, but still approximately linearly. 


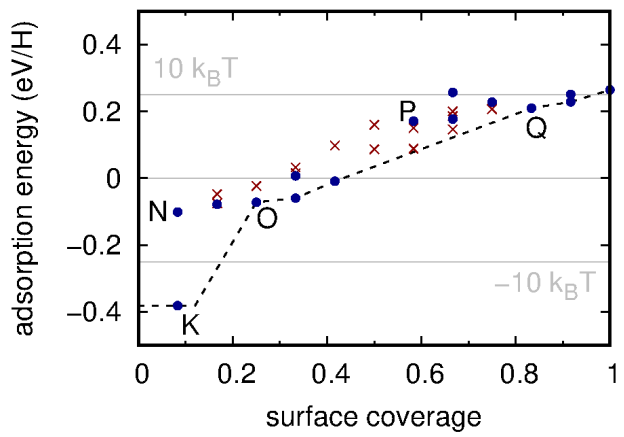

FIG. 9. Normalized adsorption energy as a function of surface coverage of LB and fcc sites on the (110) surface for each configuration in Fig. $8(\bullet)$ and for configurations with randomly distributed hydrogen atoms $(\times)$ for comparison. Lines are intended as a guide to the eye.

\section{DISCUSSION}

A picture of near-equilibrium hydriding begins to emerge as we examine adsorption energetics on all three surfaces. On the (100) surface, the lowest energy sites are H sites, which are 1-2 $k_{B} T / \mathrm{H}$ higher in energy than bulk $\mathrm{O}$ sites. Accordingly, the two-state model results suggest that the fraction of populated $\mathrm{H}$ sites will be comparable to the bulk absorption, $\mathcal{O}\left(10^{-3}\right)$. The (111) surface will have no substantial surface coverage since the lowest energy structures are all $>7 k_{B} T / \mathrm{H}$ higher in energy. On the (110) surface, adsorption in isolated $\mathrm{LB}$ sites is $-15 k_{B} T / \mathrm{H}$ relative to the bulk, suggesting that some LB sites will always be filled. Even with the majority of LB sites filled the adsorption energy increases to only $-3 k_{B} T / \mathrm{H}$. The two-state model, in turn, shows that surface sites with $-3 k_{B} T$ adsorption energies will be only be $10 \%$ filled, though this picture is somewhat oversimplified. Regardless, LB sites, though few in number due to the high surface energy of the (110) surface, could serve as nucleation sites for equilibrium hydriding due to their high stability once occupied.

We can also develop a different picture for non-equilibirum hydriding at millibar $\mathrm{H}_{2}$ pressures by comparing our adsorption energy studies to previously published values of $\mathrm{H}_{2}$ molecular adsorption and dissociation and $\mathrm{H}$ ion subsurface penetration on the (100) and (111) surfaces. Published data of this type is not available for the (110) surface and hence we limit our discussion to these two surfaces. On (100), an adsorbed $\mathrm{H}_{2}$ molecule is held to the surface only weakly, $0.15 \mathrm{eV}\left(6 k_{B} T\right) .{ }^{3}$ Computed dissociation energy barriers vary, 
$0.22 \mathrm{eV}\left(9 k_{B} T\right)^{6}$ to $0.49 \mathrm{eV}\left(19 k_{B} T\right)^{13}$ to $0.78 \mathrm{eV}\left(30 k_{B} T\right){ }^{3}$ but are all higher than the desorption barrier, suggesting that hydrogen molecules desorb and re-adsorb many times before dissociating. Once dissociated, the barrier to recombine is $1.44 \mathrm{eV}\left(56 k_{B} T\right)$, while the energy barrier for a surface hydrogen atom to burrow into a subsurface $\mathrm{T}$ site is 0.93 $\mathrm{eV}\left(36 k_{B} T\right) \cdot{ }^{13}$ Comparing the barriers for burrowing and recombination suggests, in turn, that surface hydrogen atoms are more likely to move into the bulk than recombine into an $\mathrm{H}_{2}$ molecule.

A similar but distinct picture emerges for the (111) surface. For an adsorbed $\mathrm{H}_{2}$ molecule to detach from the surface requires $0.13 \mathrm{eV}\left(5 k_{B} T\right) .{ }^{4}$ Dissociation may require less energy, $0.06 \mathrm{eV}\left(2 k_{B} T\right)^{13}$, or comparable energy, to $0.12 \mathrm{eV}\left(5 k_{B} T\right)^{6}$ to $0.31 \mathrm{eV}\left(12 k_{B} T\right) .{ }^{4}$ Either way, both desorption and dissociation can be considered barrierless. Once dissociated, the recombination barrier is $1.36 \mathrm{eV}\left(53 k_{B} T\right) \cdot{ }^{13}$ For comparison, the energy barrier for a surface hydrogen atom to burrow into the subsurface layer is half, $0.66 \mathrm{eV}\left(26 k_{B} T\right) \cdot{ }^{13}$ Moreover, the high adsorption energies computed in this work provide a high driving force for diffusion into the bulk, which is possible from both fcc and hep sites. Overall, the (111) is a likely candidate for non-equilibrium hydriding since dissociation is barrierless and there are many pathways to subsurface diffusion.

\section{CONCLUSION}

We have evaluated the surface energies of the low index facets of $\delta$-Pu. We find that the lowest energy surfaces have anti-ferromagnetic geometries with magnetic spins alternating along planes parallel to the surface. Of these, the (111) surface has the lowest surface energy and so would be the dominant surface of a macroscopic single crystal material. Polycrystalline materials will exhibit multiple surfaces; we predict the (111) will be the most abundant and the (110) the least abundant.

We have also evaluated hydrogen adsorption energies ranging from low to high surface coverage on each surface. We find that the (111) surface is the least attractive to adsorbed hydrogen, while the (110) surface is the most attractive. This suggests two mechanism for hydriding: equilibrium hydriding nucleated by the most attractive LB sites and non-equilibrium hydriding facilitated by barrierless dissociation and high driving forces for subsurface diffusion on the (111) surface. 
Future model development of plutonium surfaces needs to account for restructuring of surface atoms and the presence of surface defects present in real plutonium. Additionally, subsurface absorption energies as a function of depth below the surface, particularly the number of layers required before absorption energies are equal to bulk values, will further our understanding of the role that surface and subsurface effects play in near-equilibrium hydriding. Overall, our results yields a relatively simple description of the onset of hydriding in $\delta$ - $\mathrm{Pu}$ that can be used to provide constraints for macro-scale models of material aging and degradation. Our efforts can be extended to include interactions with plutonium oxide surfaces, which could yield advances in the way $\mathrm{Pu}$-containing devices are designed in future applications.

\section{SUPPLEMENTARY MATERIAL}

See supplementary material for the following information:

- tabulated simulation results for figures $2,4,6,8$

- additional figure showing spin ordering in AFM $\delta$-Pu slabs

\section{ACKNOWLEDGMENTS}

This work performed under the auspices of the U.S. Department of Energy by Lawrence Livermore National Laboratory under Contract DE-AC52-07NA27344. The assigned release number is LLNL-JRNL-823460-DRAFT.

\section{REFERENCES}

${ }^{1}$ https://en.wikipedia.org/wiki/Plutonium-238 (accessed Feb. 20, 2020).

${ }^{2}$ L. Dinh, J. Haschke, C. Saw, P. Allen, and W. McLean II, "Pu2o3 and the plutonium hydriding process," J. Nucl. Mater. 408, 171-175 (2011).

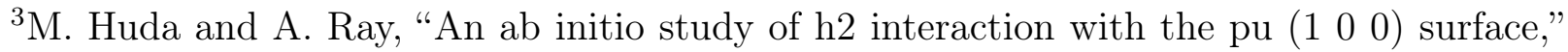
Physica B 366, 95-109 (2005).

${ }^{4}$ M. Huda and A. Ray, "Molecular hydrogen adsorption and dissociation on the plutonium (111) surface," Phys. Rev. B 72, 085101 (2005). 
${ }^{5}$ C. D. Taylor, S. C. Hernandez, M. F. Francis, D. S. Schwartz, and A. K. Ray, "Hydrogen trapping in $\delta$-pu: insights from electronic structure calculations," J. Phys. Condens. Matter 25, 265001 (2013).

${ }^{6} \mathrm{~N}$. Goldman and M. A. Morales, "A first-principles study of hydrogen diffusivity and dissociation on $\delta$-Pu (100) and (111) surfaces," J. Phys. Chem. C 121, 17950-17957 (2017).

${ }^{7}$ A. Ray and J. Boettger, "First-principles electronic structure study of the quantum size effects in (111) films of $\delta$-plutonium," Phys. Rev. B 70, 085418 (2004).

${ }^{8} \mathrm{X}$. Wu and A. K. Ray, "Full-potential lapw electronic structure study of $\delta$-plutonium and the (001) surface," Phys. Rev. B 72, 045115 (2005).

${ }^{9}$ J. Lashley, A. Lawson, R. McQueeney, and G. Lander, "Absence of magnetic moments in plutonium," Phys. Rev. B 72, 054416 (2005).

${ }^{10}$ S. C. Hernandez, D. S. Schwartz, C. D. Taylor, and A. K. Ray, "Ab initio study of gallium stabilized $\delta$-plutonium alloys and hydrogen-vacancy complexes," J. Phys. Condens. Matter 26, 235502 (2014).

${ }^{11}$ M. Huda and A. Ray, "A density functional study of atomic hydrogen adsorption on plutonium layers," Physica B 352, 5-17 (2004).

${ }^{12}$ S. C. Hernandez, S. Richmond, T. J. Venhaus, and M. N. Huda, "Influence of ga on h reactivity with ga-stabilized $\delta$-pu alloys," J. Phys. Chem. C 121, 19162-19172 (2017).

${ }^{13}$ N. Goldman, B. Aradi, R. K. Lindsey, and L. E. Fried, "Development of a multicenter density functional tight binding model for plutonium surface hydriding," J. Chem. Theory Comput. 14, 2652-2660 (2018).

${ }^{14}$ R. G. Mullen and G. Nir, "Quantum accurate prediction of plutonium-plutonium dihydride phase equilibrium using a lattice gas model," J. Phys. Chem. C (2020).

${ }^{15}$ J. L. Stakebake, "Kinetics for the reaction of hydrogen with plutonium powder," J. Alloys Compd. 187, 271-283 (1992).

${ }^{16}$ J. L. Stakebake, "Kinetics for the reaction of hydrogen with a plutonium-1 weight percent gallium alloy powder," Journal of the Electrochemical Society 128, 2383 (1981).

${ }^{17}$ J. M. Haschke and T. H. Allen, "Plutonium hydride, sesquioxide and monoxide monohydride: pyrophoricity and catalysis of plutonium corrosion," J. Alloys Compd. 320, 58-71 (2001).

${ }^{18}$ G. W. McGillivray, J. P. Knowles, I. M. Findlay, and M. J. Dawes, "The plutonium/hydrogen reaction: The pressure dependence of reaction initiation time and nu- 
cleation rate controlled by a plutonium dioxide over-layer," J. Nucl. Mater. 412, 35-40 (2011).

${ }^{19}$ A. H. Tavakoli, P. S. Maram, S. J. Widgeon, J. Rufner, K. van Benthem, S. Ushakov, S. Sen, and A. Navrotsky, "Amorphous alumina nanoparticles: structure, surface energy, and thermodynamic phase stability," J. Phys. Chem. C 117, 17123-17130 (2013).

${ }^{20}$ S. Y. Savrasov, G. Kotliar, and E. Abrahams, "Correlated electrons in $\delta$-plutonium within a dynamical mean-field picture," Nature 410, 793 (2001).

${ }^{21}$ P. Söderlind, A. Landa, and B. Sadigh, "Density-functional investigation of magnetism in $\delta$-Pu," Phys. Rev. B 66, 205109 (2002).

${ }^{22} \mathrm{P}$. Söderlind and B. Sadigh, "Density-functional calculations of $\alpha, \beta, \gamma, \delta$, $\delta$ ', and $\epsilon$ plutonium," Phys. Rev. Lett. 92, 185702 (2004).

${ }^{23} \mathrm{P}$. Söderlind, "Quantifying the importance of orbital over spin correlations in $\delta$-Pu within density-functional theory," Phys. Rev. B 77, 085101 (2008).

${ }^{24}$ T. Liu, T. Cai, T. Gao, and G. Li, "The electronic and structural properties of $\delta$-Pu and $\mathrm{PuO}$ from the LSDA (GGA)+ U method," Physica B 405, 3717-3721 (2010).

${ }^{25} \mathrm{H}$. Wang and K. Konashi, "Lda $+\mathrm{u}$ study of pu and puo2 on ground state with spin-orbital coupling," J. Alloys Compd. 533, 53-57 (2012).

${ }^{26}$ P. Söderlind, F. Zhou, A. Landa, and J. Klepeis, "Phonon and magnetic structure in $\delta$-plutonium from density-functional theory," Sci. Rep. 5, 15958 (2015).

${ }^{27}$ A. Migliori, P. Söderlind, A. Landa, F. J. Freibert, B. Maiorov, B. Ramshaw, and J. B. Betts, "Origin of the multiple configurations that drive the response of $\delta$-plutonium's elastic moduli to temperature," P. Natl. Acad. Sci. 113, 11158-11161 (2016).

${ }^{28}$ B. Ao, X. Wang, P. Shi, P. Chen, X. Ye, X. Lai, and T. Gao, "First-principles LDA+U calculations investigating the lattice contraction of face-centered cubic Pu hydrides," J. Nucl. Mater. 424, 183-189 (2012).

${ }^{29}$ G. Yong, A. Juan-Juan, G. Tao, and A. Bing-Yun, "Structural, magnetic, electronic, and elastic properties of face-centered cubic $\mathrm{PuHx}(\mathrm{x}=2,3)$ : GGA(LSDA $)+\mathrm{U}+\mathrm{SO}$," Chin. Phys. B 22, 057103 (2013).

${ }^{30}$ J.-J. Zheng, B.-T. Wang, I. Di Marco, and W.-D. Li, "Electronic structure and phase stability of plutonium hydrides: Role of coulomb repulsion and spin-orbital coupling," Int. J. Hydrog. Energy 39, 13255-13265 (2014).

${ }^{31}$ Y. Yang and P. Zhang, "Hydriding and dehydriding energies of PuHx from ab initio cal- 
culations," Phys. Lett. A 379, 1649-1653 (2015).

${ }^{32}$ G. Jomard, B. Amadon, F. Bottin, and M. Torrent, "Structural, thermodynamic, and electronic properties of plutonium oxides from first principles," Phys. Rev. B 78, 075125 (2008).

${ }^{33}$ H. Nakamura, M. Machida, and M. Kato, "Effects of spin-orbit coupling and strong correlation on the paramagnetic insulating state in plutonium dioxides," Phys. Rev. B 82, $155131(2010)$.

${ }^{34}$ G. Kresse and J. Hafner, "Ab initio molecular dynamics for liquid metals," Phys. Rev. B 47, 558 (1993).

${ }^{35}$ G. Kresse and J. Hafner, "Ab initio molecular-dynamics simulation of the liquid-metalamorphous-semiconductor transition in germanium," Phys. Rev. B 49, 14251 (1994).

${ }^{36}$ G. Kresse and J. Furthmüller, "Efficient iterative schemes for ab initio total-energy calculations using a plane-wave basis set," Phys. Rev. B 54, 11169 (1996).

${ }^{37}$ P. E. Blöchl, "Projector augmented-wave method," Phys. Rev. B 50, 17953 (1994).

${ }^{38}$ G. Kresse and D. Joubert, "From ultrasoft pseudopotentials to the projector augmentedwave method," Phys. Rev. B 59, 1758 (1999).

${ }^{39}$ J. P. Perdew, K. Burke, and M. Ernzerhof, "Generalized gradient approximation made simple," Phys. Rev. Lett. 77, 3865 (1996).

${ }^{40} \mathrm{M}$. Methfessel and A. T. Paxton, "High-precision sampling for Brillouin-zone integration in metals," Phys. Rev. B 40, 3616 (1989).

${ }^{41}$ H. J. Monkhorst and J. D. Pack, "Special points for Brillouin-zone integrations," Phys. Rev. B 13, 5188 (1976).

${ }^{42}$ S. L. Dudarev, G. A. Botton, S. Y. Savrasov, C. J. Humphreys, and A. P. Sutton, "Electron-energy-loss spectra and the structural stability of nickel oxide: An LSDA $+\mathrm{U}$ study," Phys. Rev. B 57, 1505 (1998). 